\title{
Efficient Estimation of Indirect Effects in Case-Control Studies Using a Unified Likelihood Framework
}

Running Title: Case-control mediation analysis

Glen A. Satten ${ }^{1, \#, ~ S a r a h ~ W . ~ C u r t i s ~}{ }^{2, \#, ~ C l a u d i a ~ S o l i s-L e m u s ³, ~ E l i z a b e t h ~ J . ~ L e s l i e ², ~}$ Michael P. Epstein ${ }^{2}$

\#Contributed equally to manuscript

${ }^{1}$ Department of Gynecology and Obstetrics, Emory University, Atlanta, GA

${ }^{2}$ Department of Human Genetics, Emory University, Atlanta, GA

${ }^{3}$ Department of Plant Pathology, Wisconsin Institute for Discovery, University of Wisconsin, Madison, WI

Address for Correspondence:

Michael P. Epstein, Ph.D.

Department of Human Genetics

Emory University School of Medicine

615 Michael Street, Suite 301

Atlanta, GA 30322

Phone: (404)712-8289

Email: mpepste@emory.edu 


\begin{abstract}
Mediation models are a set of statistical techniques that investigate the mechanisms that produce an observed relationship between an exposure variable and an outcome variable in order to deduce the extent to which the relationship is influenced by intermediate mediator variables. For a case-control study, the most common mediation analysis strategy employs a counterfactual framework that permits estimation of indirect and direct effects on the odds ratio scale for dichotomous outcomes, assuming either binary or continuous mediators. While this framework has become an important tool for mediation analysis, we demonstrate that we can embed this approach in a unified likelihood framework for mediation analysis in case-control studies that leverages more features of the data (in particular, the relationship between exposure and mediator) to improve efficiency of indirect effect estimates. One important feature of our likelihood approach is that it naturally incorporates cases within the exposure-mediator model to improve efficiency. Our approach does not require knowledge of disease prevalence and can model confounders and exposure-mediator interactions, and is straightforward to implement in standard statistical software. We illustrate our approach using both simulated data and real data from a case-control genetic study of lung cancer.
\end{abstract}




\section{INTRODUCTION}

Mediation techniques ${ }^{1-4}$ are indispensable epidemiological tools for exploring the relationship between an exposure of interest and an outcome variable by investigating how their relationship is affected by intermediate mediator variables. Mediation models partition the total effect that an exposure has on outcome into the indirect effect on outcome explained by the mediator and the direct effect on outcome not explained by the mediator. Classic methods for mediation analysis are embedded within a structural equation modeling (SEM) framework $^{5-7}$ that fit 3 distinct regression models: 1) regression of outcome on exposure, 2) regression of mediator on exposure, and 3) regression of outcome on exposure and mediator. Estimates of direct effect can be based on exposureoutcome relationship in model (3), while estimates of indirect effects are derived from the product of the exposure-mediator relationship in (2) and the mediatoroutcome relationship in (3) (or, alternatively, the difference of the exposureoutcome relationship in (1) and the exposure-outcome relationship in (3)). Counterfactual approaches for mediation analysis ${ }^{8-10}$ expanded on the SEM framework to allow for estimates of indirect and direct effects in the presence of potential mediator-exposure interactions as well as nonlinear relationships between variables.

Many studies, particularly in genetic epidemiology, are case-control studies. Mediation analysis for case-control studies uses the influential framework of VanderWeele (VW) and colleagues. The VW framework, published in a pair of landmark papers ${ }^{10 ; 11}$ with over 1100 citations combined, showed how the 
counterfactual framework could be used to estimate indirect and direct effects on the odds ratio scale for dichotomous outcomes assuming either binary and/or continuous mediators $10 ; 12$.

As described in Materials and Methods, the VW framework conducts inference using two separate regression models: (a) a logistic regression model of disease on exposure and mediator and (b) a separate regression model of mediator regressed on exposure. Estimates and standard errors of total, direct, and indirect effects are then derived based on parameter estimates and standard errors from these two regression models. We note that, while fitting model (a) is straightforward, fitting model (b) is problematic because the sample is nonrandomly ascertained under a case-control design, so that parameter estimates based on the study based on data from study participants do not reflect those from the population in general. To circumvent this, Valeri and VanderWeele ${ }^{10}$ used only controls to fit model (b). While this is valid, the exclusion of cases from model (b) means the VW approach loses efficiency relative to methods that could properly model the cases (accounting for ascertainment) when investigating the mediatorexposure relationship. An alternative strategy to incorporate cases into the mediator model was considered by VanderWeele et al. ${ }^{13}$, which employed weighted regression techniques that utilized inverse-probability weighting (IPW) with robust standard errors. A drawback of the IPW approach however is that the method requires knowledge of disease prevalence, which might be difficult to specify, especially when disease prevalence differs between subpopulation groups. 
In this article, we propose a likelihood approach for mediation analysis in case-control studies that provides efficiency gains over traditional VW methods. Rather than estimating indirect and direct effects based on two separate regression models, our likelihood approach jointly models the disease, exposure, and mediator together in a unified framework that accounts for correlation in the parameter estimates between the two VW regression models to provide refined estimates of these quantities. Our approach also naturally incorporates cases within the mediator-exposure model and (unlike IPW methods) does not require knowledge of disease prevalence to do so. Our method can also handle confounders and exposuremediator interactions. The method is simple to implement within the $\mathrm{R}$ programming language and we provide code (see Web Resources) for public use. Using simulated data, we show our approach provides more efficient estimates of indirect effects compared to VW approaches (including IPW strategies) for both continuous and binary mediators. We further illustrate our method using genetic and smoking data from a case-control study of lung cancer.

\section{MATERIALS AND METHODS}

Assumptions and Notation: We assume a case-control study. Define $Y$ as disease outcome ( $1=$ case, $0=$ control), $A$ as an exposure of interest (continuous or categorical), $M$ as a possible mediator (continuous or categorical), and $\boldsymbol{C}$ as a vector of confounders not influenced by exposure. We wish to fit the mediation model in Figure 1. In particular, we wish to measure the direct effect of $A$ on $Y$ and the indirect effect of $A$ on $Y$ carried out through the mediator $M$. 
VanderWeele Framework: The original VanderWeele (VW) technique ${ }^{10 ; 11}$ estimates direct and indirect effects in Figure 1 based on a formal causal inference framework 8 ; 9 . This framework conceptualizes counterfactual and potential outcomes, which define the mechanistic process by which an exposure may causally affect the dependent variable conditional on mediators. Assume a binary exposure and let $Y(a)$ be the potential outcome for exposure level $a(a=0,1)$. Then the total effect of $A$ on $Y$ is defined as $E[Y(1)]-E[Y(0)]$, which is the difference between the expectation of $Y$ when $A$ equals 1 and when $A$ equals 0 . The framework can estimate this difference despite only one of the potential outcomes being observable for a given subject, provided four assumptions hold: (I) no unmeasured exposureoutcome confounding, (II) no unmeasured mediator-outcome confounding, (III) no unmeasured exposure-mediator confounding, and (IV) no mediator-outcome confounder affected by exposure.

Under these four assumptions, we can decompose the total effect (TE) of exposure on outcome into the natural indirect effect $(N I E)$ and the natural direct effect $(N D E) . N I E$ is defined as $N I E=E\left[Y\left(1, M_{1}\right)\right]-E\left[Y\left(1, M_{0}\right)\right]$, where $M_{0}\left(M_{1}\right)$ is the counterfactual value of the mediator $M$ when $A$ equals 0 (1). Likewise, $N D E$ is defined as $N D E=E\left[Y\left(1, M_{0}\right)\right]-E\left[Y\left(0, M_{0}\right)\right]$. Thus, the total effect equals $T E=$ $N I E+N D E=E\left[Y\left(1, M_{1}\right)\right]-E\left[Y\left(0, M_{0}\right)\right]$. Additionally, for a given (fixed) value of mediator $M$, we can define the controlled direct effect $(C D E)$ as $C D E=E[Y(1, M)]-$ $E[Y(0, M)]$, which can change for different values of $M$ (as we will show). 
For case-control studies with a binary outcome, the VW techniques ${ }^{11}$ extended the definitions of $N I E, N D E$, and $C D E$ to the odds ratio scale. On this scale, the total effect (conditional on confounders $C$ ) is defined as

$$
O R^{T E}=\frac{P(Y(1)=1 \mid C) / P(Y(1)=0 \mid C)}{P(Y(0)=1 \mid C) / P(Y(0)=0 \mid C)}
$$

where $P(Y(a)=1 \mid C)$ is the probability of disease when $A=a$. TE can be partitioned into the product of the NIE and NDE, which are defined as

$$
\begin{aligned}
& O R^{N I E}=\frac{P\left(Y\left(1, M_{1}\right)=1 \mid C\right) / P\left(Y\left(1, M_{1}\right)=0 \mid C\right)}{P\left(Y\left(1, M_{0}\right)=1 \mid C\right) / P\left(Y\left(1, M_{0}\right)=0 \mid C\right)} \\
& O R^{N D E}=\frac{P\left(Y\left(1, M_{0}\right)=1 \mid C\right) / P\left(Y\left(1, M_{0}\right)=0 \mid C\right)}{P\left(Y\left(0, M_{0}\right)=1 \mid C\right) / P\left(Y\left(0, M_{0}\right)=0 \mid C\right)}
\end{aligned}
$$

The VW framework estimates $O R^{T E}, O R^{N D E}, O R^{N I E}$ by first fitting regression models to the observed data. To model the disease data, the VW framework applies the logistic model

$$
\operatorname{logit}\{P(Y=1 \mid A=a, M=m, C=c)\}=\gamma_{0}+\gamma_{A} a+\gamma_{M} m+\gamma_{A M} a m+\gamma_{C} c
$$

To model the mediator (continuous or binary), VW uses one of the following regression models

$$
\begin{aligned}
& \text { Continuous: } E[M \mid A=a, C=c]=\beta_{0}+\beta_{\mathrm{A}} a+\beta_{\mathrm{C}} c \\
& \text { Binary: } \operatorname{logit}[P(M=1 \mid A=a, C=c)]=\beta_{0}+\beta_{\mathrm{A}} a+\beta_{\mathrm{C}} c
\end{aligned}
$$


For a continuous mediator, the VW framework estimates $N I E, N D E$, and CDE on disease outcome going from exposure level $a^{*}$ to $a$ (assuming assumptions I-IV above hold) as

$$
\begin{gathered}
\log \left\{O R^{C D E}\right\}=\left(\gamma_{A}+\gamma_{A M} m\right)\left(a-a^{*}\right) \\
\log \left\{O R^{N D E}\right\} \cong\left(\gamma_{A}+\gamma_{A M}\left(\beta_{0}+\beta_{A} a^{*}+\beta_{C} c+\gamma_{M} \sigma^{2}\right)\right)\left(a-a^{*}\right)+0.5 \gamma_{A M}^{2} \sigma^{2}\left(a^{2}-a^{* 2}\right) \\
\log \left\{O R^{N I E}\right\} \cong\left(\gamma_{M} \beta_{M}+\gamma_{A M} \beta_{A} a\right)\left(a-a^{*}\right)
\end{gathered}
$$

where $\sigma^{2}$ in equation (2b) is the residual variance from fitting model (1b), which is assumed to be normally distributed. Note that $C D E$ in (2a) depends on level of the mediator (as previously mentioned). In contrast, for a binary mediator, the VW framework estimates $N I E, N D E$, and $C D E$ on disease outcome going from exposure level $a^{*}$ to $a$ as

$$
\begin{gathered}
O R^{C D E}=\exp \left\{\left(\gamma_{A}+\gamma_{A M} m\right)\left(a-a^{*}\right)\right\} \quad(3 a) \\
O R^{N D E} \cong \frac{\exp \left(\gamma_{A} a\right)\left\{1+\exp \left(\gamma_{M}+\gamma_{A M} a+\beta_{0}+\beta_{A} a^{*}+\beta_{C} c\right)\right\}}{\exp \left(\gamma_{A} a^{*}\right)\left\{1+\exp \left(\gamma_{M}+\gamma_{A M} a^{*}+\beta_{0}+\beta_{A} a^{*}+\beta_{C} c\right)\right\}} \quad(3 b) \\
O R^{N I E} \cong \frac{\left\{1+\exp \left(\beta_{0}+\beta_{A} a^{*}+\beta_{C} c\right)\right\}\left\{1+\exp \left(\gamma_{M}+\gamma_{A M} a+\beta_{0}+\beta_{A} a+\beta_{C} c\right)\right\}}{\left\{1+\exp \left(\beta_{0}+\beta_{A} a+\beta_{C} c\right)\right\}\left\{1+\exp \left(\gamma_{M}+\gamma_{A M} a+\beta_{0}+\beta_{A} a^{*}+\beta_{C} c\right)\right\}}
\end{gathered}
$$

Once we estimate these quantities, we can derive the standard errors of $O R^{T E}, O R^{N D E}, O R^{N I E}$ on the log scale by applying the delta method using the formulae outlined in the Appendix of Valeri and VanderWeele ${ }^{10}$. We can then perform hypothesis testing of $O R^{T E}, O R^{N D E}, O R^{N I E}$ using Wald tests.

The VW framework's estimation of $N D E, N I E$, and $C D E$ for both continuous mediators in (2) and binary mediators in (3) requires parameter estimates from the mediator models in (1b) and (1c). Fitting models (1b) and (1c) is difficult because 
the sample is ascertained based on disease and does not represent a random sample from a population as assumed by $E[M \mid a, c]$ in $(1 \mathrm{~b})$ and $\operatorname{logit}[P(M=1 \mid a, c)]$ in $(1 \mathrm{c})$. To circumvent this complication, VW models often make a rare-disease assumption and fit $E[M \mid a, c]$ and $\operatorname{logit}[P(M=1 \mid a, c)]$ in (1b) and (1c) using control data only. Alternatively, as in VanderWeele et al.13, one can instead incorporate cases into the mediator models in (1b) and (1c) using IPW such that cases are utilized in the model but their contributions (compared to controls) are downweighted relative to their sampling proportion. For IPW regression, the standard errors of the coefficients in (1b) and (1c) are then derived using robust Huber-White procedures ${ }^{14 ;}$.

Unified Likelihood Approach: Here, we propose a unified likelihood approach that jointly models disease, exposure, and mediator data together while incorporating cases into the mediator model accounting for ascertainment, using the rare disease approximation. Letting $j$ index subject and assuming all subjects are independent, we initially define the joint (prospective) likelihood as $L_{P}=$ $\prod_{j} P\left[Y_{j}, M_{j} \mid A_{j}, C_{j}\right]$, which we then factor into

$$
L_{P}=\prod_{j} P\left[M_{j}=m \mid Y_{j}=y, A_{j}=a, C_{j}=c\right] P\left[Y_{j}=y \mid A_{j}=a, C_{j}=c\right]
$$

We note that we could replace $P\left[Y_{j}=y \mid A_{j}=a, C_{j}=c\right]$ in (4) with $P\left[A_{j}=\right.$ $\left.a \mid Y_{j}=y, C_{j}=c\right]$ and instead perform inference using the retrospective likelihood $L_{R}=\prod_{j} P\left[A_{j}, M_{j} \mid Y_{j}, C_{j}\right]$. However, the prospective likelihood is often preferred to the retrospective likelihood as $Y \mid A, C$ in the former likelihood is easier to model (e.g. using logistic regression) than $A \mid Y, C$ in the latter likelihood; standard results ${ }^{16}$ 
assure that $L_{R}$ and $L_{P}$ are proportional if a nonparametric distribution is chosen for $A \mid C$, and that only the intercept is affected.

We first describe how to model $P\left[M_{j}=m \mid Y_{j}=y, A_{j}=a, C_{j}=c\right]$ in (4). For controls, we assume a rare disease such that $E\left[M_{j}=m \mid Y_{j}=0, A_{j}=a, C_{j}=c\right] \cong$ $\beta_{0}+\beta_{A} a+\beta_{C} c$ (for a continuous mediator) or $\operatorname{logit}\left[P\left(M=1 \mid Y_{j}=0, A_{j}=a, C_{j}=\right.\right.$ $c)]=\beta_{0}+\beta_{\mathrm{A}} a+\beta_{\mathrm{C}} c$ (for a binary mediator). These choices are the same as usually used when using the VW framework.

Data from cases can be incorporated into the mediation model; these contributions are discarded in the VW framework. To accomplish this, note that we can write $P\left[M_{j}=m \mid Y_{j}=1, A_{j}=a, C_{j}=c\right]$ as $^{17 ; 18}$

$$
P\left[M_{j}=m \mid Y_{j}=1, A_{j}=a, C_{j}=c\right]=\frac{\theta_{j}(a, m, c) P\left[M_{j}=m \mid Y_{j}=0, a, c\right]}{\int \theta_{j}\left(a, m^{*}, c\right) P\left[M_{j}=m^{*} \mid Y_{j}=0, a, c\right] d m^{*}}
$$

where

$$
\begin{aligned}
\theta_{j}(a, m, c)= & \frac{P\left[Y_{j}=1 \mid A_{j}=a, M_{j}=m, C_{j}=c\right]}{P\left[Y_{j}=0 \mid A_{j}=a, M_{j}=m, C_{j}=c\right]} \\
& =\exp \left(\gamma_{0}+\gamma_{A} a+\gamma_{M} m+\gamma_{A M} a m+\gamma_{C} c\right)
\end{aligned}
$$

is the disease odds given exposure, mediator, and confounders (allowing for possible mediator-exposure interaction, if desired). Note that $\theta_{j}(a, m, c)$ is the same quantity fit in equation (1a) in our summary of the VW framework. 
Equation (5) shows that we can naturally model the mediator in the cases (accounting for ascertainment) as a function of the disease odds in (6) and the mediator model in the controls, without requiring external information like disease prevalence (which IPW methods require). The denominator in (5) corresponds to the disease odds given exposure and confounders ${ }^{17}$; 18 . That is,

$$
\theta_{j}(a, c)=\frac{P\left[Y_{j}=1 \mid a, c\right]}{P\left[Y_{j}=0 \mid a, c\right]}=\int \theta_{j}\left(a, m^{*}, c\right) P\left[M_{j}=m^{*} \mid Y_{j}=0, a, c\right] d m^{*}
$$

which becomes

$$
=e^{\gamma_{0}+\gamma_{A} a+\gamma_{C} c} \int e^{\left(\gamma_{M}+\gamma_{A M} a\right) m^{*}} P\left[M_{j}=m^{*} \mid Y_{j}=0, a, c\right] d m^{*}
$$

if the model in equation (6) is used. $\theta_{j}(a, c)$ in (7) has a closed form whenever the moment generating function for $P\left[M_{j}=m^{*} \mid Y_{j}=0, a, c\right]$ has a simple or closed form, which simplifies inference. For example, if the mediator $M$ is normally distributed in controls with mean $E\left[M_{j}=m \mid A_{j}=a, C_{j}=c, Y_{j}=0\right]=\beta_{0}+\beta_{A} a+\beta_{C} c$ and variance $\sigma^{2}$, then we show in Supplementary Materials that $\theta_{j}(a, c)=\exp \left(\gamma_{0}+\gamma_{A} a+\right.$ $\left.\left(\gamma_{M}+\gamma_{A M} a\right)\left(\beta_{0}+\beta_{A} a+\beta_{C} c\right)+0.5\left(\gamma_{M}+\gamma_{A M} a\right)^{2} \sigma^{2}+\gamma_{C} c\right)$. For a binary $M$, we simply replace the integral in equation (7) with a sum over the two levels of the mediator.

To complete construction of $L_{P}$ in (4), we model $P\left[Y_{j}=y \mid A_{j}=a, C_{j}=c\right]$ using a logistic regression model based on $\theta_{j}(a, c)$ in $(7)$, such that $P\left[Y_{j}=\right.$ $\left.y \mid A_{j}=a, C_{j}=c\right]=\frac{\theta(a, c)^{y}}{1+\theta(a, c)}$ with the assurance that the only difference between 
using $L_{P}$ and $L_{R}$ for case-control sampling is in the intercept ${ }^{16} \gamma_{0}$. Combining all the pieces, we can simplify our likelihood for inference in (4) to give

$$
L_{P}=\prod_{j} P\left[M_{j}=m \mid Y_{j}=0, A_{j}=a, C_{j}=c\right] \frac{\theta(a, m, c)^{Y_{j}}}{1+\theta(a, c)}
$$

We propose to base inference on $L_{P}$ in (8); parameter estimates can be obtained by maximizing (8) with respect to $\left(\gamma_{0}, \gamma_{A}, \gamma_{M}, \gamma_{A M}, \gamma_{C}, \beta_{0}, \beta_{A}, \beta_{C}, \sigma^{2}\right)$ using standard optimization algorithms like Quasi-Newton, and the covariance matrix of the estimated parameters can be obtained either from the information matrix or a 'sandwich' variance estimator. We can then substitute these estimates into equations $(2 a-2 c)$ or $(3 a-3 c)$ to obtain estimates of $O R^{T E}, O R^{N D E}, O R^{N I E}$, and $O R^{C D E}$ and use the delta method results given in the Appendix of Valeri and VanderWeele ${ }^{10}$ to obtain standard errors and construct confidence intervals of these quantities. We can perform hypothesis testing of the parameters or measures of direct and indirect effects using Wald statistics based on the estimates and standard errors.

Simulation Studies: We performed simulation studies to evaluate the efficiency gains of our likelihood for mediation analysis relative to the original VW method. We assumed a continuous exposure $A$ that followed a standard normal distribution and a binary covariate $C$ that followed a Bernoulli(0.5) distribution. Using $A$ and $C$, we generated a continuous mediator $M$ using equation (1b) or generated a binary mediator $M$ using equation (1c) using parameter values shown in Table 1 . We then used $M, A, C$ to generate disease status using equation (1a) and 
parameter values shown in Table 1, assuming a value for the intercept that yielded a population prevalence of 0.03 . As shown in Table 1 , we considered models that explicitly assumed an exposure-mediator interaction as well as models that assumed no interactions. We chose parameter values to yield models with $\log \left(O R^{T E}\right)=0.145, \log \left(O R^{N D E}\right)=0.1$, and $\log \left(O R^{N I E}\right)=0.045$ for both a continuous and binary mediator, with and without a mediator-exposure interaction effect. For a specific model, we prospectively generated subjects until we obtained 300 cases and 300 controls. For all simulation settings, we generated 1000 replicates per setting.

For each simulation analysis, we compared the performance of our likelihood approach both to the original VW method that discards cases within the mediator models of (1b) and (1c) as well as the IPW-version of VW that incorporates cases into the mediator models of (1b) and (1c) using inverse probability weighting. For the IPW analyses, we assumed the disease prevalence of the weighting is correctly specified. All analyses were conducted in the R programming language. We fit our likelihood to simulated datasets using a quasi-Newton algorithm implemented in the R 'optim' function. We fit the VW methods using the R functions 'glm' and 'Im'. For the IPW method, we constructed robust Huber-White standard errors of the mediator model using the 'vcovHC' function in the R library 'sandwich'.

Application to Case-Control Genetic Study of Lung Cancer: Lung cancer is the leading cause of cancer-related deaths across the world ${ }^{19}$ and represents a major public health concern. A well-known environmental risk factor for lung cancer is tobacco smoking, with long-term smokers having a 10-fold increased risk of lung 
cancer relative to non-smokers ${ }^{20}$. Beyond smoking and other environmental risk factors, genetics are also known to broadly play a role in lung-cancer risk $^{21 ; 22}$ with several specific risk loci being identified within genome-wide association studies (GWAS) of the disease $23 ; 24$.

One established risk loci of lung cancer identified by GWAS resides on chromosome $15 q 25.1^{25}$. The associated region contains nicotinic acetylcholine receptor subunit genes that encode proteins that form receptors that bind nicotine. A number of studies suggest that this risk locus is also associated with measures of smoking behavior ${ }^{26-29}$. Given the known relationship between smoking behavior and lung cancer, it is worth inquiring whether the SNPs in this locus influence lung cancer risk partially or completely through smoking behavior. To explore these hypotheses, we utilized genetic, smoking pack-year, and disease data from the GENEVA GWAS of Lung Cancer and Smoking ${ }^{30} 31$ available through dbGaP (accession number phs000093.v2.p2; see Web Resources). The GENEVA GWAS dataset consists of 2695 cases and 2779 controls genotyped for 508,916 post-QC SNPs across the genome.

We first used the GENEVA data to perform a genome-wide analysis of the post-QC autosomal SNPs with lung cancer using logistic regression, adjusted for gender and the top three principal components of ancestry (Manhattan and quantile-quantile plots are shown in Figures S1 and S2, respectively). Within the chromosome 15q25.1 locus, we identified strong associations of several SNPs with lung cancer in GENEVA with SNP rs12914385 ( $\left.p=1.887 \times 10^{-15}\right)$ yielding the top signal in the region (see Figure S3). We next conducted a GWAS of a dichotomized version 
of smoking pack-years (split at the median value of 3 pack-years) in the GENEVA data using logistic regression, adjusting for gender and the top 3 principal components of ancestry (Manhattan and quantile-quantile plots are shown in Figures S4 and S5, respectively). Interestingly, we observed the same lung cancer risk SNP rs12914385 also yielded a strong association with the dichotomized packyear variable $\left(p=1.364 \times 10^{-6}\right)$ and likewise was the top signal in the chromosome 15q25.1 locus (see Figure S6).

Based on our results, we explored the relationship of rs12914385 with smoking pack-years and lung cancer within a mediation framework. Specifically, we were interested in exploring how much this SNP (exposure $A$ ) influenced lung cancer disease status (outcome $Y$ ) through the dichotomized smoking pack-year variable (mediator $M$ ). Our analyses also controlled for gender and the top three principal components of ancestry (covariates $C$ ). Within controls, the minor-allele frequency of $r s 12914385$ was 0.402 . We performed inference using both our likelihood approach as well as traditional VW techniques.

We note that VanderWeele and colleagues ${ }^{13} ; 32$ previously conducted similar analyses to untangle the relationship between SNPs in 15q25.1 locus, smoking, and lung cancer using case-control mediation tools. Our analysis above differs from these previous analyses in a few important ways. First, the previous analysis did not use the GENEVA study and instead analyzed a different collection of genetic studies of lung cancer. Second, the authors did not consider rs12914385 as the SNP exposure and instead studied two nearby SNPs (rs8034191, rs1051730) in moderate linkage disequilibrium with rs12914385. Finally, they also considered different 
measures of smoking than pack-years and did not adjust for principal components of ancestry as we do in our analyses presented here.

\section{RESULTS}

Simulations with Continuous Mediator: Table 2 provides simulation results for estimating and conducting inference of $O R^{T E}, O R^{N D E}, O R^{N I E}, O R^{C D E}$ using our likelihood approach, the original VW method, and the VW-IPW under the generating model that assumed a continuous mediator with no mediator-exposure interaction effect on disease risk. We noticed important differences among the methods with regards to inference of indirect effects (highlighted in Table 2). In particular, the simulation results revealed that our likelihood approach yielded smaller (but still well calibrated) standard errors of the indirect effects, relative to the VW approach, due in part to the former method incorporating cases within the mediation model that the latter method discards. Consequently, Table 2 shows our likelihood approach has greater power to detect the indirect effect relative to the VW method. We performed additional power simulations under a wider range of indirect-effect size estimates (generated by varying the value of $\gamma_{M}$ in (2c) while holding the remaining simulation parameters at the values shown in Table 1) and observed that the likelihood approach was substantially more powerful than the VW method for detecting an indirect effect across all values considered (Figure 2). For total and direct effects, the likelihood, VW, and VW-IPW methods all yielded similar findings.

Our likelihood approach improves efficiency of indirect effects by incorporating cases within the mediation model. This impact can be illuminated by 
studying the standard errors of the mediator-model parameter estimates (see Table S1), which are much smaller for our likelihood approach than for the VW approach. Additionally, by jointly modeling disease, mediator, and exposure data in a unified framework, we account for correlations among the disease-model and mediatormodel parameters (see Table S2); the VW framework assumes no correlation among parameters from these two different models. By incorporating these non-zero correlations in our likelihood framework, our approach refines the standard errors of the indirect effects estimated using the delta method. Results using the VW-IPW approach (which incorporates cases but substantially downweights their contribution relative to controls) were quite similar to those using the VW approach but did yield slightly smaller standard errors for mediator-model parameter estimates relative to VW.

We next considered simulation models with a continuous mediator where we generated a mediator-exposure interaction. Table 3 shows estimates and standard errors for $O R^{T E}, O R^{N D E}, O R^{N I E}, O R^{C D E}$ when we assume an interaction effect. For indirect effects, the VW and VW-IPW methods had larger mean estimates of standard error than the empirical standard deviation of the effect estimates and likewise had wider coverage for the $95 \%$ CI than expected $(98.0 \%$ for both the VW and VW-IPW methods). Our likelihood approach, on the other hand, had appropriate coverage of the $95 \%$ confidence interval for the indirect effect and the mean estimates of standard error mirrored the empirical standard deviation of estimates in the simulated datasets. Consequently, the likelihood approach had increased power to detect the indirect effect relative to the VW and VW-IPW techniques. The 
likelihood, VW, and VW-IPW methods all yielded similar inference for total and direct effects when an interaction effect was incorporated in the model.

To understand the improved power of our likelihood approach for detecting indirect effects, inspection of the parameter estimates (Table S3) show that the likelihood approach provides more efficient estimates of the mediator-exposure interaction effect in the disease model, as well as those parameters related to the mediator model. Inspection of the mean covariance estimates for model parameters (Table S4) shows the likelihood approach estimates non-zero correlations between disease-model parameters (including the mediator-exposure interactions) and mediator-model parameters that the VW and IPW-VW methods presume are 0.

Simulations with Binary Mediator: Table 4 provides estimates and standard errors for $O R^{T E}, O R^{N D E}, O R^{N I E}, O R^{C D E}$ for a binary mediator assuming no mediatorexposure interaction effect. For $O R^{N I E}$, we observed that the likelihood approach yielded smaller standard errors than the VW and VW-IPW approaches and consequently had increased power to detect this indirect effect. We subsequently conducted additional power simulations under a broader range of indirect-effect size estimates (generated by varying values of $\gamma_{M}$ in (3c) while holding the remaining simulation parameters at the values shown in Table 1) and observed substantially increased power for the likelihood approach over the VW method across this range (Figure 3). The likelihood approach, VW, and VW-IPW approaches all produced similar results for total and direct effects.

Inspection of parameter estimates (Table S5) and mean parameter covariance (Table S6) reveal similar trends to what we observed in the analysis of 
continuous mediators; the likelihood approach produces mediator-model parameter estimates with smaller standard errors than the VW and VW-IPW methods and the likelihood approach estimates non-zero covariances between disease-model parameters and mediator-model parameters.

We finally consider simulation models with a binary mediator assuming a mediator-exposure interaction effect on disease risk and show the main results in Table 5. For the indirect effect, our likelihood approach had appropriate coverage of the $95 \%$ confidence interval with mean standard errors for the effect estimates that mirrored the empirical standard deviation of the parameter estimates in the simulated datasets. The VW and VW-IPW methods yielded unbiased estimates for the effects as well but the coverage of the $95 \%$ confidence interval for the indirect effects was larger than expected (98.6\% for both the VW and VW-IPW methods) and the mean standard errors of the indirect effects was larger than the empirical standard deviation of the parameter estimates produced by the methods across the simulated datasets. Consequently, the likelihood approach had markedly increased power to detect the indirect effect relative to the VW/VW-IPW methods. Inspection of model parameter estimates (Table S7) and mean parameter covariance (Table S8) revealed similar findings as previously observed for other simulation models. Results for direct and total effects were similar among all three methods when we modeled an interaction effect.

Application to Case-Control Genetic Study of Lung Cancer: Using the 2695 cases and 2779 controls from the GENEVA GWAS of lung cancer, we first conducted a mediation analysis that assessed whether the significant effect of SNP rs12914385 
on lung cancer risk was due in part to an indirect effect through a mediator of smoking pack-years, adjusting for covariate effects of gender and the top three principal components of ancestry. We initially applied our likelihood approach, as well as the VW approach, to the data explicitly assuming a potential interaction effect $\gamma_{A M}$ of exposure (SNP genotype) and mediator (smoking pack-years) on disease risk. However, we observed no significant interaction effect using either our likelihood approach $(p=0.147)$ or the VW approach $(p=0.130)$. Thus, we removed the interaction parameter from each model and proceeded with reduced models that assumed no interaction effects.

Under the reduced models, we provide the odds-ratio estimates of indirect and direct effects of rs12914385 on lung-cancer risk using our likelihood in Table 6 (see Table S9 for corresponding parameter estimates produced using our approach). Overall, our results demonstrate that the effect of rs12914385 on lung cancer risk is predominantly a direct effect but there is evidence of a modest indirect effect through smoking pack-years. Specifically, the total effect of rs12914385 on lung cancer risk $\left(O R^{T E}=1.358 ; 95 \% \mathrm{CI}:[1.255,1.470]\right)$ is nearly completely due to a direct effect $\left(O R^{N D E}=1.333 ; 95 \%\right.$ CI: $\left.[1.226,1.449]\right)$ of the SNP on disease outcome rather than an indirect effect through the mediator of smoking pack-years $\left(O R^{N I E}=1.019 ; 95 \% \mathrm{CI}:[1.000,1.039]\right)$. That being said, the test of the indirect effect was significant ( $p=0.0494)$ using our approach; additional analyses using larger sample sizes to explore the indirect effect further is warranted.

We next repeated the same mediation analysis of $r s 129143851$ using the traditional VW approach (Table 6, Table S9). While the estimates of total, direct, and 
indirect effects using the VW approach were quite similar to those produced using our likelihood approach, we observed that the VW method's confidence intervals were noticeably wider both for the total effect as well as the indirect effect compared to our likelihood approach. Furthermore, the VW approach found no borderline evidence of an indirect effect of $r$ 129143851 on lung-cancer risk through smoking pack-years $(p=0.5204)$. Inspection of parameter estimates (see Supplementary Table 9) revealed the likelihood approach yielded smaller standard errors for parameter estimates within the mediation model than the VW approach, mirroring our observations within our simulated datasets.

\section{DISCUSSION}

Our work develops a unified likelihood approach for case-control mediation analysis that improves inference of indirect effects relative to the popular counterfactual framework of VanderWeele by jointly modeling the disease model and mediator model together in a joint framework that further leverages important information on the mediator-exposure relationship within cases. Existing VW methods either ignore cases in the mediator model or use IPW procedures to incorporate them into the model. Unlike our approach, IPW requires knowledge of disease prevalence which may be difficult to ascertain. Even if prevalence information is correctly specified, the IPW-VW techniques fit the disease model and mediator model separately and so does not account for the correlation among parameters between the two models. Our likelihood framework utilizes this correlation, thereby resulting in more efficient estimates of parameters within the 
mediator model compared to the VW models. We also note our framework also leads to more efficient estimates of the mediator-exposure interaction variable in the disease model when modeled. Due to the increased efficiency of these parameter estimates (as well as accounting for parameter correlation between disease and mediator models), we also subsequently obtain more efficient estimates of indirect effects between an exposure and disease outcome compared to the VW framework. Our method further does not require knowledge of disease prevalence to incorporate cases into our framework.

We illustrated our method using genetic data from a GWAS study of lung cancer and found that the effect of the top risk SNP in the chromosome 15q25.1 locus on lung cancer within this dataset is predominantly a direct effect but there is some evidence for a modest indirect effect through smoking pack-years. While we applied our unified likelihood within a case-control genetics project for illustration purposes, the technique is widely applicable in other settings (e.g. environmental studies, psychological studies) that employ a case-control sampling design. We provide R code implementing the likelihood approach on our website (see Web Resources). Extension of our framework to handle multiple mediators is feasible and will be explored in future work.

Both our likelihood approach and the VW framework model the probability of disease conditional on exposure and mediator even though, by design, casecontrol studies generate retrospectively ascertained mediator and exposure data. Such prospective analysis can be as efficient as a retrospective analysis but this result holds only under the assumption of a saturated non-parametric distribution 
for the exposure ${ }^{16}$. Carroll ${ }^{33}$ showed that prospective analysis of retrospectivelysampled data may be less efficient than a retrospective analysis when one restricts the exposure distribution in some fashion. For example, genetic studies often restrict the distribution of exposure (genotype or haplotype) by making the logical assumption that the underlying alleles are in Hardy-Weinberg Equilibrium (HWE). In these situations, many studies have shown ${ }^{34-37}$ that retrospective analysis of casecontrol genetic data can be more efficient than prospective analysis under the HWE assumption. Thus, in future work, we will investigate the use of a retrospective likelihood for case-control mediation analysis that may improve on prospective approaches by directly modeling the distribution of exposure conditional on outcome.

\section{ACKNOWLEDGEMENTS}

This work was supported by NIH grants DE029698 and GM117946.

\section{WEB RESOURCES}

dbGaP site for GENEVA study: https://www.ncbi.nlm.nih.gov/projects/gap/cgibin/study.cgi?study_id=phs000093.v2.p2

Epstein Software: https://sites.google.com/view/epsteinlab/software 


\section{BIBLIOGRAPHY}

1. MacKinnon, D. (2012). Introduction to statistical mediation analysis. (Routledge).

2. MacKinnon, D.P. (2011). Integrating mediators and moderators in research design. Research on social work practice 21,675_681.

3. VanderWeele, T.J. (2016). Mediation analysis: a practitioners guide. Annual review of public health $37,17-32$.

4. Richiardi, L., Bellocco, R., and Zugna, D. (2013). Mediation analysis in epidemiology: methods, interpretation and bias. International journal of epidemiology 42, 1511-1519.

5. Baron, R. M., and Kenny, D.A. (1986). The moderator-mediator variable distinction in social psychological research: Conceptual, strategic, and statistical considerations. Journal of personality and social psychology 51 , 1173.

6. Judd, C. M., and Kenny, D.A. (1981). Process analysis: Estimating mediation in treatment evaluations. Evaluation review 5, 602-619.

7. Sobel, M.E. (1982). Asymptotic confidence intervals for indirect effects in structural equation models. Sociological methodology 13, 290_312.

8. Pearl, J. (2001). Direct and indirect effects. In Proceedings of the seventeenth conference on uncertainty in artificial intelligence. (Morgan Kaufmann Publishers Inc. ), pp 411_420.

9. Robins, J. M., and Greenland, S. (1992). Identifiability and exchangeability for direct and indirect effects. Epidemiology, 143-155.

10. Valeri, L., and VanderWeele, T. J. (2013). Mediation analysis allowing for exposure-mediator interactions and causal interpretation: theoretical assumptions and implementation with SAS and SPSS macros. Psychological methods 18, 137.

11. VanderWeele, T. J. , and Vansteelandt, S. (2010). Odds ratios for mediation analysis for a dichotomous outcome. American journal of epidemiology 172, 1339_1348.

12. VanderWeele, T., and Vansteelandt, S. (2014). Mediation analysis with multiple mediators. Epidemiologic methods 2, 95-115.

13. VanderWeele, T. J., Asomaning, K., Tchetgen Tchetgen, E. J., Han, Y., Spitz, M. R., Shete, S., Wu, X., Gaborieau, V., Wang, Y., and McLaughlin, J. (2012). Genetic 
variants on 15q25. 1, smoking, and lung cancer: an assessment of mediation and interaction. American journal of epidemiology 175, 1013_1020.

14. White, H. (1980). A heteroskedasticity_consistent covariance matrix estimator and a direct test for heteroskedasticity. Econometrica: journal of the Econometric Society, 817_838.

15. Huber, P. J. (1967). The behavior of maximum likelihood estimates under nonstandard conditions. In Proceedings of the fifth Berkeley symposium on mathematical statistics and probability. (University of California Press ), pp 221-233.

16. Prentice, R. L. , and Pyke, R. (1979). Logistic disease incidence models and casecontrol studies. Biometrika 66, 403_411.

17. Satten, G.A., and Kupper, L. L. (1993 ). Inferences about exposure_disease associations using probability_of_exposure information. Journal of the American Statistical Association 88, 200_208.

18. Satten, G. A. , and Carroll, R. J. (2000 ). Conditional and unconditional categorical regression models with missing covariates. Biometrics 56, 384-388.

19. Siegel, R., Ma, J., Zou, Z., and Jemal, A. (2014 ). Cancer statistics, 2014. CA: a cancer journal for clinicians 64, 9-29.

20. Humans, I. W. G. o.t.E. o. C. R.t., Organization, W. H. , and Cancer, I. A.f. R. o. (2004). Tobacco smoke and involuntary smoking. ( Iarc).

21. Jonsson, S., Thorsteinsdottir, U., Gudbjartsson, D. F., Jonsson, H. H., Kristjansson, K. , Arnason, S., Gudnason, V., Isaksson, H. J., Hallgrimsson, J. , and Gulcher, J.R. (2004). Familial risk of lung carcinoma in the Icelandic population. Jama 292, 2977_2983.

22. Mucci, L. A., Hjelmborg, J. B., Harris, J. R., Czene, K., Havelick, D. J., Scheike, T., Graff, R.E., Holst, K. , Möller, S. , and Unger, R. H. (2016). Familial risk and heritability of cancer among twins in Nordic countries. Jama 315, 68_76.

23. McKay, J. D., Hung, R. J., Han, Y., Zong, X., Carreras_Torres, R. , Christiani, D. C., Caporaso, N.E., Johansson, M. , Xiao, X., and Li, Y. (2017). Large_scale association analysis identifies new lung cancer susceptibility loci and heterogeneity in genetic susceptibility across histological subtypes. Nature genetics 49, 1126-1132.

24. Bossé, Y., and Amos, C.I. (2018). A decade of GWAS results in lung cancer. Cancer Epidemiology and Prevention Biomarkers 27, 363_379.

25. Hung, R. J., McKay, J. D., Gaborieau, V., Boffetta, P., Hashibe, M., Zaridze, D. , Mukeria, A., Szeszenia_Dabrowska, N., Lissowska, J., and Rudnai, P. (2008). 
A susceptibility locus for lung cancer maps to nicotinic acetylcholine receptor subunit genes on 15q25. Nature 452, 633-637.

26. Freathy, R. M., Ring, S. M., Shields, B., Galobardes, B., Knight, B., Weedon, M. N., Smith, G. D. , Frayling, T. M., and Hattersley, A. T. (2009). A common genetic variant in the 15q24 nicotinic acetylcholine receptor gene cluster (CHRNA5CHRNA3-CHRNB4 ) is associated with a reduced ability of women to quit smoking in pregnancy. Human molecular genetics 18, 2922_2927.

27. Munafò, M. R., Johnstone, E. C., Walther, D. , Uhl, G. R., Murphy, M. F. , and Aveyard, P. (2011). CHRNA3 rs1051730 genotype and short-term smoking cessation. Nicotine \& Tobacco Research 13,982_988.

28. Saccone, N. L., Culverhouse, R. C., Schwantes_An, T._H., Cannon, D. S., Chen, X., Cichon, S., Giegling, I., Han, S., Han, Y., and Keskitalo_Vuokko, K. ( 2010 ). Multiple independent loci at chromosome 15q25. 1 affect smoking quantity: a meta-analysis and comparison with lung cancer and COPD. PLoS Genet 6, e1001053.

29. Weiss, R. B., Baker, T. B., Cannon, D. S., Von Niederhausern, A., Dunn, D. M., Matsunami, N., Singh, N. A., Baird, L., Coon, H. , and McMahon, W. M. (2008). A candidate gene approach identifies the CHRNA5_A3_B4 region as a risk factor for age_dependent nicotine addiction. PLoS Genet 4, e1000125.

30. Prorok, P. C., Andriole, G. L. , Bresalier, R. S., Buys, S. S., Chia, D. , Crawford, E. D., Fogel, R., Gelmann, E. P., Gilbert, F., and Hasson, M. A. (2000). Design of the

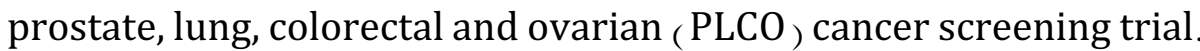
Controlled clinical trials 21, 273S_309S.

31. Landi, M. T., Consonni, D., Rotunno, M., Bergen, A. W., Goldstein, A. M. , Lubin, J. H., Goldin, L., Alavanja, M., Morgan, G., and Subar, A. F. (2008). Environment And Genetics in Lung cancer Etiology (EAGLE) study: an integrative population_based case_control study of lung cancer. BMC public health 8,1-10.

32. VanderWeele, T. J. (2013). A three-way decomposition of a total effect into direct, indirect, and interactive effects. Epidemiology (Cambridge, Mass ) 24, 224.

33. Carroll, R., Wang, S., and Wang, C. (1995). Prospective analysis of logistic casecontrol studies. Journal of the American Statistical Association 90, 157-169.

34. Epstein, M.P., and Satten, G.A. (2003). Inference on haplotype effects in casecontrol studies using unphased genotype data. The American Journal of Human Genetics 73, 1316-1329. 
35. Kwee, L., Epstein, M., Manatunga, A., Duncan, R., Allen, A., and Satten, G. (2007). Simple methods for assessing haplotype - environment interactions in case - only and case - control studies. Genetic Epidemiology: The Official Publication of the International Genetic Epidemiology Society 31, 75_90.

36. Satten, G. A., and Epstein, M.P. (2004). Comparison of prospective and retrospective methods for haplotype inference in case - control studies. Genetic Epidemiology: The Official Publication of the International Genetic Epidemiology Society 27, 192_201.

37. Chen, J., and Chatterjee, N. (2007). Exploiting Hardy_Weinberg equilibrium for efficient screening of single SNP associations from case_control studies. Human heredity 63, 196. 


\section{TABLE 1: SIMULATION MODELS}

\begin{tabular}{|c|c|c|c|c|}
\hline & \multicolumn{2}{|c|}{ Continuous Mediator } & \multicolumn{2}{c|}{ Binary Mediator } \\
\hline & No Interaction & Interaction & No Interaction & Interaction \\
\hline & & & & \\
\hline$\gamma_{A}$ & 0.100 & 0.077 & 0.100 & 0.049 \\
\hline$\gamma_{M}$ & 0.300 & 0.200 & 0.420 & 0.320 \\
\hline$\gamma_{A M}$ & $\mathrm{X}$ & 0.100 & $\mathrm{X}$ & 0.100 \\
\hline$\gamma_{C}$ & 0.050 & 0.050 & 0.100 & 0.100 \\
\hline$\beta_{0}$ & 0.100 & 0.100 & -0.310 & -0.310 \\
\hline$\beta_{A}$ & 0.150 & 0.200 & 0.430 & 0.430 \\
\hline$\beta_{C}$ & 0.050 & 0.050 & 0.100 & 0.100 \\
\hline$\sigma^{2}$ & 0.500 & 0.500 & $\mathrm{X}$ & $\mathrm{X}$ \\
\hline & & & & \\
\hline $\log \left(O R^{T E}\right)$ & 0.145 & 0.145 & 0.145 & 0.145 \\
\hline $\log \left(O R^{N D E}\right)$ & 0.100 & 0.100 & 0.100 & 0.100 \\
\hline $\log \left(O R^{N I E}\right)$ & 0.045 & 0.045 & 0.045 & 0.045 \\
\hline $\log \left(O R^{C D E}\right)$ & 0.100 & 0.077 & 0.100 & 0.049 \\
\hline
\end{tabular}

$O R^{T E}, O R^{N D E}, O R^{N I E}$, and $O R^{C D E}$ calculated where $a^{*}=0, a=1, c=0$, and $m=0$ 
TABLE 2: CONTINUOUS MEDIATOR WITH NO INTERACTION

\begin{tabular}{|c|c|c|c|c|c|c|c|c|}
\hline & & & & & & \multicolumn{3}{|c|}{ Power at $\alpha=$} \\
\hline & $\begin{array}{c}\text { True } \\
\text { Value }\end{array}$ & $\begin{array}{c}\text { Mean } \\
\text { Value }\end{array}$ & $\begin{array}{c}\text { Mean } \\
\text { SE }\end{array}$ & SD & $\begin{array}{c}\text { 95\% CI } \\
\text { Coverage }\end{array}$ & 0.05 & 0.01 & 0.001 \\
\hline Likelihood & & & & & & & & \\
\hline $\log \left(O R^{T E}\right)$ & $\mathbf{0 . 1 4 5}$ & 0.144 & 0.083 & 0.081 & 0.961 & 0.408 & 0.198 & 0.055 \\
\hline $\log \left(O R^{N D E}\right)$ & $\mathbf{0 . 1 0 0}$ & 0.099 & 0.085 & 0.083 & 0.958 & 0.199 & 0.072 & 0.014 \\
\hline $\log \left(O R^{N I E}\right)$ & $\mathbf{0 . 0 4 5}$ & 0.045 & 0.019 & 0.019 & 0.956 & 0.691 & 0.362 & 0.058 \\
\hline $\log \left(O R^{C D E}\right)$ & $\mathbf{0 . 1 0 0}$ & 0.099 & 0.085 & 0.083 & 0.958 & 0.199 & 0.072 & 0.014 \\
\hline & & & & & & & & \\
\hline VW & & & & & & & & \\
\hline $\log \left(O R^{T E}\right)$ & $\mathbf{0 . 1 4 5}$ & 0.144 & 0.084 & 0.081 & 0.964 & 0.397 & 0.182 & 0.046 \\
\hline $\log \left(O R^{N D E}\right)$ & $\mathbf{0 . 1 0 0}$ & 0.099 & 0.085 & 0.083 & 0.958 & 0.199 & 0.072 & 0.014 \\
\hline $\log \left(O R^{N I E}\right)$ & $\mathbf{0 . 0 4 5}$ & 0.045 & 0.022 & 0.021 & 0.940 & 0.504 & 0.131 & 0.009 \\
\hline $\log \left(O R^{C D E}\right)$ & $\mathbf{0 . 1 0 0}$ & 0.099 & 0.085 & 0.083 & 0.958 & 0.199 & 0.072 & 0.014 \\
\hline & & & & & & & & \\
\hline $\mathbf{V W}$-IPW & & & & & & & & \\
\hline $\log \left(O R^{T E}\right)$ & $\mathbf{0 . 1 4 5}$ & 0.144 & 0.084 & 0.081 & 0.964 & 0.397 & 0.185 & 0.047 \\
\hline $\log \left(O R^{N D E}\right)$ & $\mathbf{0 . 1 0 0}$ & 0.099 & 0.085 & 0.083 & 0.958 & 0.199 & 0.072 & 0.014 \\
\hline $\log \left(O R^{N I E}\right)$ & $\mathbf{0 . 0 4 5}$ & 0.045 & 0.022 & 0.021 & 0.948 & 0.521 & 0.143 & 0.008 \\
\hline $\log \left(O R^{C D E}\right)$ & $\mathbf{0 . 1 0 0}$ & 0.099 & 0.085 & 0.083 & 0.958 & 0.199 & 0.072 & 0.014 \\
\hline
\end{tabular}


TABLE 3: CONTINUOUS MEDIATOR WITH INTERACTION

\begin{tabular}{|c|c|c|c|c|c|c|c|c|}
\hline & & & & & & & ver at & \\
\hline & $\begin{array}{l}\text { True } \\
\text { Value }\end{array}$ & $\begin{array}{l}\text { Mean } \\
\text { Value }\end{array}$ & Mean SE & Emp. SD & $\begin{array}{c}95 \% \text { CI } \\
\text { Coverage }\end{array}$ & 0.05 & 0.01 & 0.001 \\
\hline \multicolumn{9}{|l|}{ Likelihood } \\
\hline $\log \left(O R^{T E}\right)$ & 0.145 & 0.145 & 0.084 & 0.084 & 0.956 & 0.407 & 0.215 & 0.055 \\
\hline $\log \left(O R^{N D E}\right)$ & 0.100 & 0.103 & 0.085 & 0.086 & 0.951 & 0.240 & 0.086 & 0.018 \\
\hline $\log \left(O R^{N I E}\right)$ & 0.045 & 0.043 & 0.022 & 0.022 & 0.960 & 0.565 & 0.281 & 0.048 \\
\hline $\log \left(O R^{C D E}\right)$ & 0.077 & 0.079 & 0.087 & 0.090 & 0.940 & 0.143 & 0.057 & 0.006 \\
\hline \multicolumn{9}{|l|}{ VW } \\
\hline $\log \left(O R^{T E}\right)$ & 0.145 & 0.146 & 0.088 & 0.086 & 0.962 & 0.372 & 0.178 & 0.034 \\
\hline $\log \left(O R^{N D E}\right)$ & 0.100 & 0.104 & 0.086 & 0.087 & 0.952 & 0.238 & 0.087 & 0.020 \\
\hline $\log \left(O R^{N I E}\right)$ & 0.045 & 0.043 & 0.028 & 0.023 & 0.980 & 0.235 & 0.013 & 0.000 \\
\hline $\log \left(O R^{C D E}\right)$ & 0.077 & 0.079 & 0.088 & 0.091 & 0.941 & 0.152 & 0.054 & 0.005 \\
\hline \multicolumn{9}{|l|}{ VW-IPW } \\
\hline $\log \left(O R^{T E}\right)$ & 0.145 & 0.148 & 0.088 & 0.086 & 0.963 & 0.376 & 0.183 & 0.034 \\
\hline $\log \left(O R^{N D E}\right)$ & 0.100 & 0.104 & 0.086 & 0.087 & 0.952 & 0.239 & 0.088 & 0.020 \\
\hline $\log \left(O R^{N I E}\right)$ & 0.045 & 0.043 & 0.028 & 0.023 & 0.980 & 0.254 & 0.016 & 0.000 \\
\hline $\log \left(O R^{C D E}\right)$ & 0.077 & 0.079 & 0.088 & 0.091 & 0.941 & 0.152 & 0.054 & 0.005 \\
\hline
\end{tabular}


TABLE 4: BINARY MEDIATOR WITH NO INTERACTION

\begin{tabular}{|c|c|c|c|c|c|c|c|c|}
\hline & & & & & & \multicolumn{3}{|c|}{ Power at $\alpha=$} \\
\hline & $\begin{array}{c}\text { True } \\
\text { Value }\end{array}$ & $\begin{array}{c}\text { Mean } \\
\text { Value }\end{array}$ & $\begin{array}{c}\text { Mean } \\
\text { SE }\end{array}$ & SD & $\begin{array}{c}\text { 95\% CI } \\
\text { Coverage }\end{array}$ & 0.05 & 0.01 & 0.001 \\
\hline Likelihood & & & & & & & & \\
\hline $\log \left(O R^{T E}\right)$ & $\mathbf{0 . 1 4 5}$ & 0.142 & 0.083 & 0.083 & 0.953 & 0.391 & 0.193 & 0.061 \\
\hline $\log \left(O R^{N D E}\right)$ & $\mathbf{0 . 1 0 0}$ & 0.097 & 0.085 & 0.085 & 0.949 & 0.200 & 0.080 & 0.021 \\
\hline $\log \left(O R^{N I E}\right)$ & $\mathbf{0 . 0 4 5}$ & 0.045 & 0.020 & 0.021 & 0.940 & 0.649 & 0.319 & 0.034 \\
\hline $\log \left(O R^{C D E}\right)$ & $\mathbf{0 . 1 0 0}$ & 0.097 & 0.085 & 0.085 & 0.949 & 0.200 & 0.080 & 0.021 \\
\hline & & & & & & & & \\
\hline VW & & & & & & & & \\
\hline $\log \left(O R^{T E}\right)$ & $\mathbf{0 . 1 4 5}$ & 0.142 & 0.084 & 0.083 & 0.961 & 0.373 & 0.184 & 0.055 \\
\hline $\log \left(O R^{N D E}\right)$ & $\mathbf{0 . 1 0 0}$ & 0.097 & 0.085 & 0.085 & 0.949 & 0.200 & 0.080 & 0.021 \\
\hline $\log \left(O R^{N I E}\right)$ & $\mathbf{0 . 0 4 5}$ & 0.045 & 0.023 & 0.024 & 0.920 & 0.497 & 0.134 & 0.008 \\
\hline $\log \left(O R^{C D E}\right)$ & $\mathbf{0 . 1 0 0}$ & 0.097 & 0.085 & 0.085 & 0.949 & 0.200 & 0.080 & 0.021 \\
\hline & & & & & & & & \\
\hline $\mathbf{V W}$-IPW & & & & & & & & \\
\hline $\log \left(O R^{T E}\right)$ & $\mathbf{0 . 1 4 5}$ & 0.142 & 0.084 & 0.083 & 0.961 & 0.374 & 0.185 & 0.055 \\
\hline $\log \left(O R^{N D E}\right)$ & $\mathbf{0 . 1 0 0}$ & 0.097 & 0.085 & 0.085 & 0.949 & 0.200 & 0.080 & 0.021 \\
\hline $\log \left(O R^{N I E}\right)$ & $\mathbf{0 . 0 4 5}$ & 0.045 & 0.022 & 0.023 & 0.923 & 0.519 & 0.153 & 0.008 \\
\hline $\log \left(O R^{C D E}\right)$ & $\mathbf{0 . 1 0 0}$ & 0.097 & 0.085 & 0.085 & 0.949 & 0.200 & 0.080 & 0.021 \\
\hline
\end{tabular}


TABLE 5: BINARY MEDIATOR WITH INTERACTION

\begin{tabular}{|c|c|c|c|c|c|c|c|c|}
\hline & & & & & & \multicolumn{3}{|c|}{ Power at $\alpha=$} \\
\hline & $\begin{array}{c}\text { True } \\
\text { Value }\end{array}$ & $\begin{array}{c}\text { Mean } \\
\text { Value }\end{array}$ & $\begin{array}{c}\text { Mean } \\
\text { SE }\end{array}$ & SD & $\begin{array}{c}\text { 95\% CI } \\
\text { Coverage }\end{array}$ & 0.05 & 0.01 & 0.001 \\
\hline Likelihood & & & & & & & & \\
\hline $\log \left(O R^{T E}\right)$ & $\mathbf{0 . 1 4 5}$ & 0.148 & 0.084 & 0.085 & 0.946 & 0.427 & 0.214 & 0.053 \\
\hline $\log \left(O R^{N D E}\right)$ & $\mathbf{0 . 1 0 0}$ & 0.107 & 0.085 & 0.084 & 0.953 & 0.243 & 0.080 & 0.023 \\
\hline $\log \left(O R^{N I E}\right)$ & $\mathbf{0 . 0 4 5}$ & 0.041 & 0.023 & 0.023 & 0.969 & 0.501 & 0.218 & 0.029 \\
\hline $\log \left(O R^{C D E}\right)$ & $\mathbf{0 . 0 4 9}$ & 0.055 & 0.117 & 0.117 & 0.953 & 0.077 & 0.018 & 0.002 \\
\hline & & & & & & & & \\
\hline VW & & & & & & & & \\
\hline $\log \left(O R^{T E}\right)$ & $\mathbf{0 . 1 4 5}$ & 0.148 & 0.088 & 0.085 & 0.961 & 0.392 & 0.168 & 0.036 \\
\hline $\log \left(O R^{N D E}\right)$ & $\mathbf{0 . 1 0 0}$ & 0.107 & 0.086 & 0.084 & 0.951 & 0.233 & 0.079 & 0.021 \\
\hline $\log \left(O R^{N I E}\right)$ & $\mathbf{0 . 0 4 5}$ & 0.041 & 0.029 & 0.023 & 0.986 & 0.193 & 0.020 & 0.000 \\
\hline $\log \left(O R^{C D E}\right)$ & $\mathbf{0 . 0 4 9}$ & 0.055 & 0.118 & 0.118 & 0.951 & 0.075 & 0.017 & 0.002 \\
\hline & & & & & & & & \\
\hline $\mathbf{V W}$-IPW & & & & & & & & \\
\hline $\log \left(O R^{T E}\right)$ & $\mathbf{0 . 1 4 5}$ & 0.149 & 0.088 & 0.085 & 0.959 & 0.392 & 0.172 & 0.037 \\
\hline $\log \left(O R^{N D E}\right)$ & $\mathbf{0 . 1 0 0}$ & 0.108 & 0.086 & 0.084 & 0.951 & 0.235 & 0.079 & 0.021 \\
\hline $\log \left(O R^{N I E}\right)$ & $\mathbf{0 . 0 4 5}$ & 0.041 & 0.029 & 0.023 & 0.986 & 0.224 & 0.020 & 0.000 \\
\hline $\log \left(O R^{C D E}\right)$ & $\mathbf{0 . 0 4 9}$ & 0.055 & 0.118 & 0.118 & 0.951 & 0.075 & 0.017 & 0.002 \\
\hline
\end{tabular}


TABLE 6: MEDIATION ANALYSIS OF rs12914385 AND LUNG CANCER

\begin{tabular}{|c|c|c|c|}
\hline & Estimate & $\begin{array}{c}95 \% \mathrm{CI} \\
\text { Coverage }\end{array}$ & P-value \\
\hline \multicolumn{4}{|l|}{ Likelihood } \\
\hline$O R^{T E}$ & 1.358 & $(1.255,1.470)$ & $<0.0001$ \\
\hline$O R^{N D E}$ & 1.333 & $(1.226,1.449)$ & $<0.0001$ \\
\hline$O R^{N I E}$ & 1.019 & $(1.000,1.039)$ & 0.0494 \\
\hline$O R^{C D E}$ & 1.333 & $(1.226,1.449)$ & $<0.0001$ \\
\hline \multicolumn{4}{|l|}{ VW } \\
\hline$O R^{T E}$ & 1.347 & $(1.232,1.474)$ & $<0.0001$ \\
\hline$O R^{N D E}$ & 1.333 & $(1.226,1.449)$ & $<0.0001$ \\
\hline$O R^{N I E}$ & 1.011 & $(0.978,1.044)$ & 0.5204 \\
\hline$O R^{C D E}$ & 1.333 & $(1.226,1.449)$ & $<0.0001$ \\
\hline
\end{tabular}


bioRxiv preprint doi: https://doi.org/10.1101/2021.07.16.452552; this version posted July 16, 2021. The copyright holder for this preprint (which was not certified by peer review) is the author/funder, who has granted bioRxiv a license to display the preprint in perpetuity. It is made available under aCC-BY-NC-ND 4.0 International license.

FIGURE 1: MEDIATION DIAGRAM

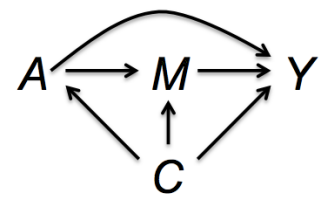




\section{FIGURE 2: POWER TO DETECT INDIRECT EFFECT (CONTINUOUS MEDIATOR)}

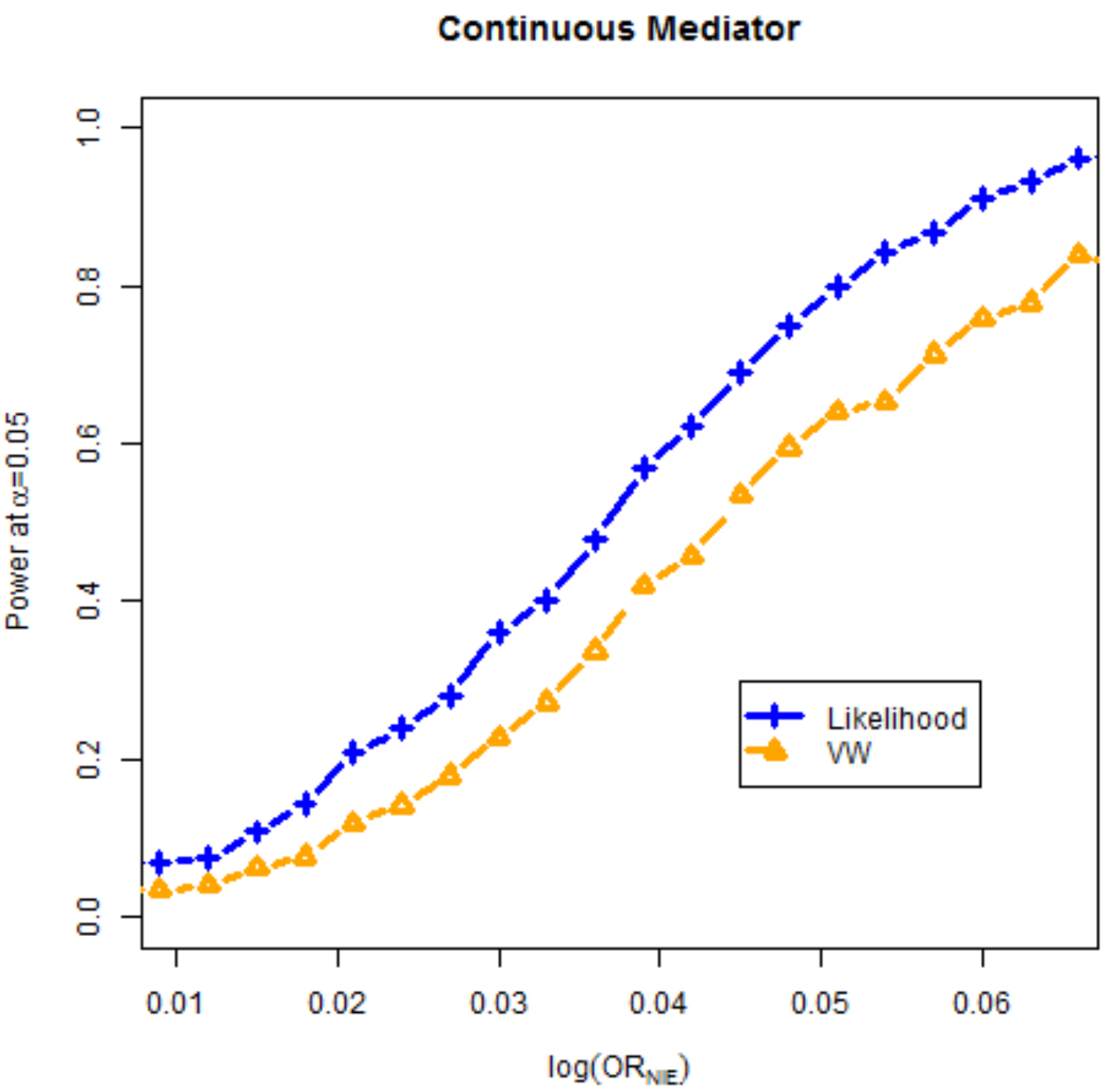

Power of likelihood framework and original VW method to detect $\log \left(O R^{N I E}\right)$ for 300 cases and 300 controls assuming continuous mediator and no exposure-mediator interaction effect. Simulation parameters shown in Table 1 for continuous mediator, with exception of $\gamma_{M}$ which is varied to produce range of values of $\log \left(O R^{N I E}\right)$ shown in x-axis of plot. 


\section{FIGURE 3: POWER TO DETECT INDIRECT EFFECT (BINARY MEDIATOR)}

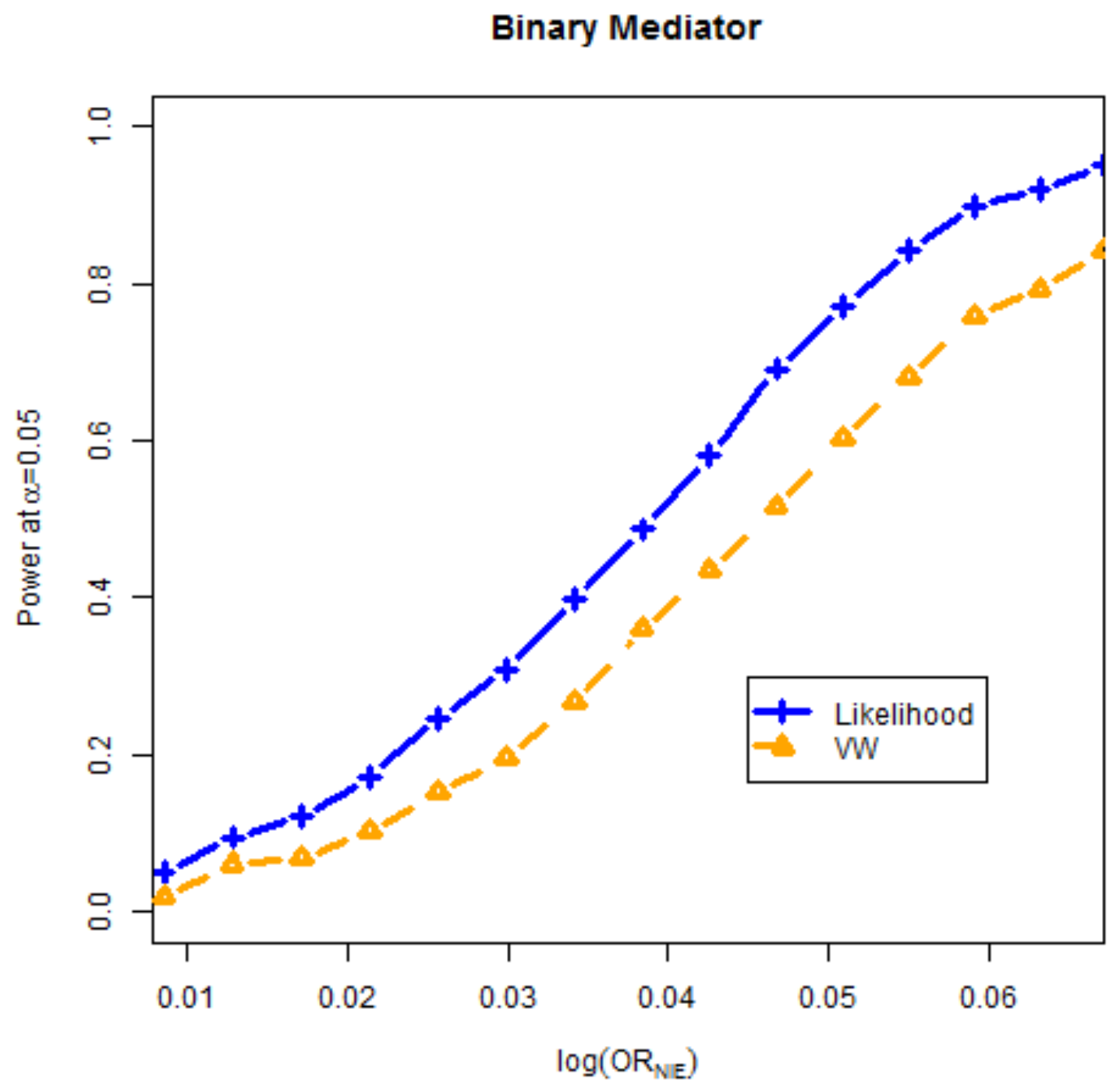

Power of likelihood framework and original VW method to detect $\log \left(O R^{N I E}\right)$ for 300 cases and 300 controls assuming binary mediator and no exposure-mediator interaction effect. Simulation parameters shown in Table 1 for binary mediator, with exception of $\gamma_{M}$ which is varied to produce range of values of $\log \left(O R^{N I E}\right)$ shown in $\mathrm{x}$-axis of plot. 